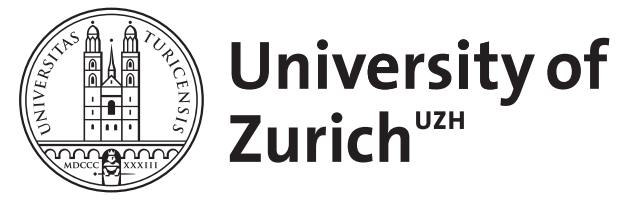

\title{
Die lokoregionale Radiotherapie verlängert beim de novo metastasierten Nasopharynxkarzinom das Überleben signifikant
}

\author{
Balermpas, Panagiotis
}

DOI: https://doi.org/10.1007/s00066-020-01715-8

Other titles: Locoregional radiotherapy in de novo metastatic nasopharyngeal carcinoma significantly prolongs survival

Posted at the Zurich Open Repository and Archive, University of Zurich ZORA URL: https://doi.org/10.5167/uzh-193871

Journal Article

Accepted Version

Originally published at:

Balermpas, Panagiotis (2021). Die lokoregionale Radiotherapie verlängert beim de novo metastasierten Nasopharynxkarzinom das Überleben signifikant. Strahlentherapie und Onkologie, 197(1):82-84.

DOI: https://doi.org/10.1007/s00066-020-01715-8 


\section{Balermpas: Literaturkommentar}

Die lokoregionale Radiotherapie verlängert beim de novo metastasierten Nasopharynxkarzinom das Überleben signifikant

Local Radiotherapy in de-novo metastatic nasopharyngeal carcinoma significantly prolongs survival

PD Dr. med. Panagiotis Balermpas, Zürich

\section{Originalpublikation}

You R, Liu YP, Huang PY et al (2020) Efficacy and Safety of Locoregional Radiotherapy With Chemotherapy vs Chemotherapy Alone in De Novo Metastatic Nasopharyngeal Carcinoma - A Multicenter Phase III Randomized Clinical Trial. JAMA Oncol doi:10.1001/jamaoncol.2020.1808

Published online July 23, 2020.

Hintergrund Eine definitive Radiotherapie (RT) oder Radiochemotherapie (RCT) ist die Standardbehandlung für das lokal begrenzte bzw. lokoregional fortgeschrittene Nasopharynxkarzinom [1]. Die Rolle einer lokalen Bestrahlung in der metastasierten Situation war aber bis jetzt nicht ausreichend untersucht und damit unklar. Ziel der vorliegenden Publikation war in erster Linie die Ermittlung des Gesamtüberlebens (OS), aber auch des progressionsfreie Überlebens (PFS) und der Toxizitätsraten in einer multizentrischen, prospektiv randomisierten Studie zwischen lokal bestrahlten und nicht bestrahlten Patienten mit metastasiertem Nasopharynxkarzinom (mNPC)

Patientengut und Methoden Insgesamt 126 von ursprünglich 173 rekrutierten Patienten mit kompletter oder partieller Remission nach 3 Zyklen Chemotherapie mit Cisplatin/ 5FU (PF) wurden 1:1 randomisiert zu maximal 6 Zyklen Systemtherapie, bestehend aus Cisplatin (100 mg/qm) und $5 \mathrm{~g} / \mathrm{qm}$ 5FU als kontinuierliche Infusion über 120 Stunden am Tag 1, oder dieselbe Behandlung, gefolgt von einer intensitätsmodulierten Radiotherapie des Primarius und der befallenen Lymphknotenregionen. Alle Patienten hatten eine histologisch gesicherte Diagnose und eine prätherapeutische lokale MRI-Bildgebung sowie ein PET-CT zur Bestätigung der Fernmetastasierung. Die Radiotherapie musste innerhalb von 21 Tagen nach der Randomisierung beginnen. Es wurden normofraktioniert 70 Gy in 33 Fraktionen am makroskopischen Primärtumor und 60-66 Gy an den befallenen Lymphknoten sowie 56-66 Gy in 
der erweiterten Tumorregion und 50-60 Gy in den befallenen zervikalen Levels appliziert.

Die Nachsorge erfolgte alle 2-3 Monate.

Ergebnisse Nach einer verblindeten Interim-Analyse im August 2018 stoppte das unabhängige Sicherheitskomitee die Studie vor Erreichen der geplanten Rekrutierung von 204 Patienten wegen der statistisch grossen Unterschiede bei den Zielkriterien. Von den 126 randomisierten Patienten mit kompletter Remission waren 83.3\% Männer und 16.7\% Frauen, das mediane Alter betrug 46 Jahre. Das 2-Jahres - Überleben war 76.4\% in der Bestrahlungsgruppe (CRT) und 54.5\% in der Gruppe mit alleiniger Chemotherapie (CT). Damit war die Studie positiv mit einem signifikanten Überlebensvorteil durch die CRT mit einer HR 0.42 (95\% Cl 0.23-0.77, p = 0.004). Ähnliche Ergebnisse zugunsten der CRT waren auch beim PFS-Endpunkt zu beobachten: HR 0.36 (95\% Cl 0.23-0.57). Die Rate an hämatologischen, gastrointestinalen, hepatischen und nephrologischen Komplikationen zeigte keine signifikanten Unterschiede zwischen den beiden Gruppen. Akute, mit der Radiotherapie assoziierte Grad 3 - Toxizitäten waren u.a. 8.1\% Dermatitis, 33.9\% Mucositis und 6.5\% Xerostomie. Als Spättoxizitäten vom Grad 3 sind am häufigsten Hörverlust (5.2\%) und Trismus (3.4\%) aufgefallen.

Schlussfolgerung der Autoren Die lokoregionale Radiotherapie nach Chemotherapie verbessert das Gesamtüberleben von Patienten mit Chemotherapie-sensitivem mNPC signifikant.

\section{Kommentar}

In den letzten Jahren konnten prospektiv-randomisierte Studien Vorteile durch eine intensivierte Chemotherapie zusätzlich zur Radiatio für nicht metastasierte Patienten demonstrieren [2]. In der metastasierten Situation dagegen bleibt die alleinige klassische Chemotherapie, bestehend aus Cisplatin und 5FU oder neuerlich Cisplatin und Gemcitabin, seit mehreren Jahren der Standard [3]. Dieser brachte allerdings keine wesentlichen Fortschritte beim Überleben dieser Patienten. Dabei ist eine Fernmetastasierung bei Nasopharynxkarzinom gar nicht so selten: $8 \%$ der Patienten werden mit synchronen Metastasen diagnostiziert, und 20\%-30\% werden im weiteren Krankheitsverlauf solche entwickeln [4]. Die Arbeit von You et al. ist die erste randomisierte Studie zum Stellenwert der Radiotherapie bei Nasopharynxkarzinomen in der metastasierten Situation und bestätigt ihren Einsatz erfolgreich. Die Toxizität war moderat und nicht unerwartet.

Frühere retrospektive Analysen haben in den letzten Jahren schon positive Daten für die lokale Bestrahlung des mNPC geliefert: Rusthoven et al. haben zum Beispiel in einer der 
größten Kohorten, bestehend aus 718 Fällen aus der Nationalen Krebs-Datenbank (NCDB), ein um 18\% verbessertes 5-Jahres-Überleben beobachtet nach einer medianen Nachbeobachtung von 4.4 Jahren nach CRT [5]. Ähnliche Ergebnisse wurden auch von Zou et al. beobachtet [6]. Diese Gruppe hatte 462 Patienten gemäß deren metastatischer Tumorlast kategorisiert und die Beobachtungen an diesem Trainingsset sogar mit internen und externen Gruppen weiter validiert. Besonders Patienten mit oligometastatischer Erkrankung und diese mit mehreren Metastasen ohne hepatischem Befall hatten eindeutig von der aggressiven lokalen Therapie profitiert. Das 3-Jahres-Überleben betrug bis zu 72.8\% für die prognostisch günstigere oligometastasierte Subgruppe. Sowohl diese retrospektiven Studien als auch die hier präsentierte randomisierte Studie haben die strenge Selektion von ausschließlich de-novo metastasierten Patienten gemeinsam. Ob sich die positiven Ergebnisse auf andere onkologische Situationen. z.B. die metachrone Metastasierung oder die induzierte Oligometastasierung oder die Oligoprogression übertragen lassen, ist bisher noch unklar. Es wäre zum Beispiel denkbar, dass die beobachteten Überlebensvorteile mehr mit der Gesamt-Tumorlast (wie in der Studie von Zou (REF)) und weniger mit dem Zeitpunkt der Metastasierung zu tun haben.

Die größte Datenbank-Analyse zum Thema, publiziert in 2017, hat insgesamt 3269 Patienten mit metastatischen Kopf-Hals Tumoren aller Stadien inkludiert und konnte einen signifikanten Überlebensvorteil nach 2 Jahren- von 13.6\% (34.2\% vs. 20.6\%) für Patienten mit radikaler Lokaltherapie (definiert als Operation oder Radiatio mit mindestens $60 \mathrm{~Gy}$ ) zeigen [7]. Die absolute Mehrheit der Patienten mit „radikaler“ Behandlung (1299 von 1495) hat eine Radiotherapie bekommen. In der Studie wurden auch 295 mNPC-Patienten mit ausgewertet. Interessanterweise, waren hier weder die Anzahl der Metastasen noch die Bestrahlungsdosis signifikante prognostische Faktoren. Patienten mit einer palliativen Dosis < 60 Gy hatten allerdings keinen Überlebensvorteil. Da diese beiden Faktoren -niedrige Tumorlast zum Zeitpunkt der Behandlung und höhere „lokal kurative“ Dosis- in allen Studien signifikant das onkologische Ergebnis beeinflussten, sollten sie auch bei der täglichen klinischen Entscheidungsfindung eine Rolle spielen. Des Weiteren hatten auch Patienten in Hoch-Volumen Zentren einen deutlicheren Überlebensvorteil, was für die Notwendigkeit einer Zentralisierung solcher schwierigen Fälle in großen, erfahrenen Tumorzentren spricht.

Die Ergebnisse der prospektiven Studie von You können noch besser vor einem allgemeinen Paradigmenwechsel in der radioonkologischen Indikationsstellung betrachtet werden, wo die radioonkologische Indikation sich im Bereich der Metastasierung immer mehr ausbreitet. Moderne technische Entwicklungen wie IMRT und SBRT haben eine schonende und effektive Behandlung dieser Patienten mit fortgeschrittenen Erkrankungen erst möglich ge- 
macht. Beeindruckende Behandlungsergebnisse konnten bis jetzt sowohl für die lokale Behandlung des Primarius, wie im Beispiel der STAMPEDE-Studie für Prostatakarzinom [8], als auch für die Behandlung der Metastasen in der Histologie-agnostischen SABR-COMET Studie [9] beobachtet werden. Das verbesserte Verständnis für die biologischen Hintergründe, aber auch für die genauere Differenzierung der verschiedenen oligometastasierten Situationen werden künftig wohl auch eine bessere Patientenselektion erlauben [10].

\section{Fazit}

Die hier besprochene Publikation bestätigt die in der radioonkologischen Praxis immer häufiger eingesetzte Strategie der lokalen hochdosierten Radiotherapie von Primärtumor und Metastasen beim primär metastasierten Nasopharynxkarzinom. Sie liefert damit zum ersten Mal Grad I - Evidenz für die entsprechende Indikationsstellung. Die radioonkologische Behandlung von metastasierten Malignomen wird parallel mit der Verbesserung internistischer Systemtherapien in der Zukunft eine immer wichtigere Rolle spielen.

Panagiotis Balermpas, Zürich

Kontaktadresse:

PD Dr. med. Dr. med. habil. Panagiotis Balermpas

Klinik für Radio-Onkologie, Universitätsspital Zürich, Rämistrasse 100, 8091 Zürich

Panagiotis.Balermpas@usz.ch

\section{Interessenskonflikt}

Panagiotis Balermpas gibt an, dass kein Interessenskonflikt besteht.

\section{Literatur}

1. Chan ATC, Grégoire V, Lefebvre J-L, et al (2012) Nasopharyngeal cancer: EHNSESMO-ESTRO Clinical Practice Guidelines for diagnosis, treatment and follow-up. Ann Oncol Off J Eur Soc Med Oncol 23 Suppl 7:vii83-85. https://doi.org/10.1093/annonc/mds266

2. Zhang Y, Sun Y, Ma J (2019) Induction gemcitabine and cisplatin in locoregionally advanced nasopharyngeal carcinoma. Cancer Commun Lond Engl 39:39. https://doi.org/10.1186/s40880-019-0385-5

3. Zhang L, Huang Y, Hong S, et al (2016) Gemcitabine plus cisplatin versus fluorouracil plus cisplatin in recurrent or metastatic nasopharyngeal carcinoma: a multicentre, randomised, open-label, phase 3 trial. Lancet Lond Engl 388:1883-1892. https://doi.org/10.1016/S0140-6736(16)31388-5 
4. Lee AW, Law SC, Ng SH, et al (1992) Retrospective analysis of nasopharyngeal carcinoma treated during 1976-1985: late complications following megavoltage irradiation. Br J Radiol 65:918-928. https://doi.org/10.1259/0007-1285-65-778-918

5. Rusthoven CG, Lanning RM, Jones BL, et al (2017) Metastatic nasopharyngeal carcinoma: Patterns of care and survival for patients receiving chemotherapy with and without local radiotherapy. Radiother Oncol J Eur Soc Ther Radiol Oncol 124:139-146. https://doi.org/10.1016/j.radonc.2017.03.019

6. Zou X, You R, Liu H, et al (2017) Establishment and validation of M1 stage subdivisions for de novo metastatic nasopharyngeal carcinoma to better predict prognosis and guide treatment. Eur J Cancer Oxf Engl 1990 77:117-126. https://doi.org/10.1016/j.ejca.2017.02.029

7. Zumsteg ZS, Luu M, Yoshida EJ, et al (2017) Combined high-intensity local treatment and systemic therapy in metastatic head and neck squamous cell carcinoma: An analysis of the National Cancer Data Base. Cancer 123:4583-4593. https://doi.org/10.1002/cncr.30933

8. Parker CC, James ND, Brawley CD, et al (2018) Radiotherapy to the primary tumour for newly diagnosed, metastatic prostate cancer (STAMPEDE): a randomised controlled phase 3 trial. Lancet Lond Engl 392:2353-2366. https://doi.org/10.1016/S01406736(18)32486-3

9. Palma DA, Olson R, Harrow S, et al (2020) Stereotactic Ablative Radiotherapy for the Comprehensive Treatment of Oligometastatic Cancers: Long-Term Results of the SABR-COMET Phase II Randomized Trial. J Clin Oncol Off J Am Soc Clin Oncol JCO2000818. https://doi.org/10.1200/JCO.20.00818

10. Guckenberger M, Lievens Y, Bouma AB, et al (2020) Characterisation and classification of oligometastatic disease: a European Society for Radiotherapy and Oncology and European Organisation for Research and Treatment of Cancer consensus recommendation. Lancet Oncol 21:e18-e28. https://doi.org/10.1016/S14702045(19)30718-1 